\title{
O que mudou no desempenho educacional dos Institutos Federais do Brasil?
}

\author{
Rogério Severiano Dutra ${ }^{a}$ \\ Giselle Bezerra Mesquita Dutra ${ }^{b}$ \\ Paulo Henrique Nobre Parente ${ }^{c}$ \\ Edilson Paulo ${ }^{d}$
}

\section{Resumo}

A pesquisa investigou os níveis diferenciados da proficiência obtida pelos Institutos Federais do Brasil no ENEM, pois se compreende que as políticas públicas adotadas nacionalmente para a Rede Federal de Ensino fortalecem ao longo do tempo a oferta de ensino. Para atendimento do objetivo proposto, a população estudada foi composta por todos os Institutos Federais do Brasil que divulgaram dados referentes à proficiência educacional obtida pelas médias das áreas do conhecimento do ENEM, por escola, extraídos do sítio eletrônico do INEP no período de 2011 a 2015. O percurso metodológico foi estabelecido por métodos estatísticos univariados de medidas de tendência central, de dispersão, de correlação e de comparação de médias entre as variáveis. Com base nos achados, verificou-se que o desempenho dos Institutos Federais do Brasil tende a ser crescente e significante no transcorrer dos anos, tanto quando avaliado como rede de ensino, quanto quando avaliado por região.

Palavras-chave: Teoria da função de produção. Desempenho educacional. Políticas públicas educacionais. ENEM.

\section{Introdução}

O desempenho, de modo geral, é considerado o resultado obtido pela combinação de fatores de entrada e de saída. Pela teoria da função de produção educacional, segundo Bowles (1970, p. 12), o desempenho educacional pode ser descrito pela "relação entre as entradas (insumos) da escola e dos estudantes e uma medida

\footnotetext{
a Instituto Federal de Educação, Ciência e Tecnologia do Ceará, Tauá, CE, Brasil.

b Secretaria de Educação do Ceará, Caucaia, CE, Brasil.

c Instituto Federal de Educação, Ciência e Tecnologia do Ceará. Acaraú, CE, Brasil.

d Universidade Federal do Rio Grande do Sul, Porto Alegre, RS, Brasil. 
de saída (rendimento acadêmico)". Dessa forma, as organizações educacionais gerenciam os recursos disponíveis com o objetivo de aprimorar constantemente seus resultados.

Censo divulgado pelo Instituto Nacional de Estudos e Pesquisas Educacionais Anísio Teixeira (INEP) aponta que o Brasil conta com 186,1 mil escolas de educação básica (INEP, 2017). O mesmo assinala que $0,4 \%$ destas estão sob a responsabilidade da União, representadas pelas escolas da Rede Federal de Ensino.

Apesar desse baixo percentual de escolas pertencentes à Rede Federal de Ensino, a mesma tem-se destacado, segundo dados do INEP. Para Ramos (2018), tal fato pode ser atribuído à qualidade do ensino ofertada por suas políticas públicas, verificada, por exemplo, pelos resultados do Exame Nacional do Ensino Médio (ENEM), os quais fornecem feedback para os estudantes e para as organizações de ensino. Os alunos que realizam os exames são concluintes da Educação Básica que se preparam para o ingresso no Ensino Superior ou para o mercado de trabalho, assim como buscam realizar a avaliação de seus conhecimentos e desenvolvimento de competências e habilidades (COTTA, 2001). Já, para as instituições de ensino, esses resultados também podem ser utilizados para realizar diagnósticos, reflexões, planejamentos e formulação de políticas públicas para aprimoramento da qualidade do ensino ofertado (RAMOS, 2018; WERLE, 2011).

O Ministério da Educação conferiu ao ENEM, a partir do ano de 2009, a dupla função de avaliar conhecimentos e de selecionar para o Ensino Superior, concedendo qualidade e status similares a avaliações como o "Exame de Maturidade", aplicado em países da Europa, e o Scholastic Assessment Test (SAT), aplicado nos Estados Unidos da América (ALMEIDA, 2018; BECHER; TOMASI; JUSTO, 2018; MENALDO, 2018). Desse modo, o ENEM é parâmetro para avaliar conhecimentos desenvolvidos pelo estudante, assim como de acesso às universidades públicas e privadas do Brasil (SLONIAK et al., 2013), servindo de base para o desenvolvimento do Ensino Básico em todo o território nacional.

Assim, o desempenho educacional, mensurado pelas avaliações em larga escala, passou a ser determinante para a implantação de políticas educacionais (RAMOS, 2018), pois, afeta os avaliados, em ambiente intra e extraescolar (TOFFOLI; SIMON, 2018). As pesquisas identificadas com a temática das avaliações em larga escala utilizam, geralmente:

I. para o Ensino Fundamental, a Prova Brasil (ROSISTOLATO; PRADO; MARTINS, 2018; SALGADO JUNIOR; NOVI, 2015); 
II. para o Ensino Médio, o Exame Nacional do Ensino Médio (ENEM) (HAGUETTE; PESSOA; VIDAL, 2016); e

III. para o Ensino Superior, o Exame Nacional de Desempenho de Estudantes (Enade) (BELTRÃO; MANDARINO, 2014; PAIVA; FREIRE; FERNANDES, 2012).

Salienta-se que o tema é pouco explorado com enfoque nos diversos níveis de ensino ofertado pelos Institutos Federais (IF) do Brasil. Portanto, comprovada a importância da avaliação em larga escala no cenário nacional como política pública, e a carência de estudos no âmbito dos IF do Brasil, o presente estudo, com foco no Ensino Básico regular, pretende avaliar a evolução e as tendências do desempenho dos Institutos Federais de Educação, Ciência e Tecnologia do Brasil, por meio dos resultados auferidos no ENEM, com uso de inferências estatísticas entre os períodos estudados, na expectativa de que os efeitos sejam significantes.

Assim, conforme a Teoria da Função de Produção, a avaliação dos resultados alcançados pelos IF do Brasil contribui com o aperfeiçoamento da aplicação de seus insumos, e, considerando que o ENEM constitui uma ferramenta periódica de avaliação que determina a proficiência dos conhecimentos, das habilidades e das competências dos estudantes e das organizações de ensino, a pesquisa assume como objetivo investigar os níveis diferenciados da proficiência obtida pelos Institutos Federais do Brasil no ENEM.

Em 2015, a Rede Federal de Ensino divulgou seus últimos resultados do Programa de Avaliação Internacional de Estudantes (PISA) e do ENEM. No primeiro, o desempenho mostrou-se superior às médias nacionais de Ciências (403 pontos), Leitura (407 pontos) e Matemática (377 pontos) alcançando, respectivamente, as médias de 517, 528 e 488 pontos (INEP, 2016). No segundo, a média geral da Rede Federal de Ensino foi de 572,42 pontos, superando as redes Estadual (492,39), Municipal $(520,40)$ e Privada $(570,41)$ (INEP, 2015).

Com base nessa informação, espera-se que esses resultados possam ter sido construídos pelas instituições de forma progressiva e significante ao longo dos anos. Desse modo, a pesquisa adota a seguinte hipótese geral $\left(\mathrm{H}_{\mathrm{g}}\right)$ : $\mathbf{O}$ desempenho, alcançado pelas instituições federais de ensino básico no transcorrer do período, é crescente e significante.

O estudo se diferencia das demais pesquisas, e pode ser considerado relevante por: (i) contribuir com a temática da avaliação do desempenho de organizações de ensino; (ii) utilizar o ENEM como métrica do desempenho educacional; (iii) 
verificar, de modo censitário, o desempenho educacional dos Institutos Federais de Educação, Ciência e Tecnologia; e (iv) considerar os resultados publicados, por escola, do exame das últimas edições.

O percurso metodológico da pesquisa seguiu com a definição da população e a aplicação de testes estatísticos. As médias, por instituição, no ENEM, publicadas na plataforma de microdados, no sítio eletrônico do INEP, foram determinantes do conjunto populacional, e tiveram como fator limitante, para o número de anos estudados, a decisão do Governo Federal de não publicar esses resultados após o ano de 2015.

O trabalho encontra-se dividido em cinco seções, incluindo esta introdução. A próxima seção trata do referencial teórico, que aborda a Teoria da Função de Produção Educacional e o Desempenho Educacional. Na terceira parte do trabalho, tem-se o delineamento metodológico. Os resultados da pesquisa, bem como as discussões acerca destes, serão apresentados no quarto tópico, seguidos das considerações finais, que serão abordadas na quinta e última seção.

\section{Referencial teórico}

\subsection{Teoria da Função de Produção Educacional}

As medidas de desempenho observadas pela Teoria de Função de Produção Educacional podem ser verificadas por meio de frequências escolares, taxas de rendimentos (aprovação, reprovação e abandono) e conclusão do Ensino Básico, ingresso no Ensino Superior, dentre outras. Entretanto, comumente, são utilizados resultados em testes padronizados de larga escala.

Assim, a função de produção educacional examina a relação de produtividade entre os insumos que afetam desempenho escolar e produto final (níveis de proficiência do aluno), combinando insumos escolares e familiares para obtenção maximizada dos resultados educacionais (TODD; WOLPIN, 2003). Corroborando com esse pensamento, Hanushek, (2003) explica que os fatores que determinam o desempenho escolar estão, geralmente, associados: à (i) estrutura escolar, à (ii) família e ao (iii) indivíduo, e, dessa forma, pode-se estimar uma função de produção educacional, em que permita que a proficiência possa ser explicada em função desses fatores.

Coleman et al. (1966) apresentam, como fatores explicativos do desempenho dos alunos, os contextos nos quais estão inseridos os insumos e os processos escolares. A mensuração dos efeitos dos fatores sobre o desempenho educacional 
possibilita estratificar e identificar características carentes da ação de políticas públicas eficientes, e que a utilização da função de produção educacional permite relacionar os fatores listados anteriormente a uma variável resposta indicadora de desempenho ou eficiência educacional (LUZ, 2006).

Desse modo, os fatores reunidos nas instituições de ensino afetam, de modo diferenciado, o desempenho educacional, que pode ser mensurado por avaliações internas, assim como externas, promovidas pelo Ministério da Educação (MEC), dentre elas, o ENEM.

\subsection{Políticas Públicas e proficiência no ENEM}

Existem três tipos de políticas públicas desenvolvidas por meio de seu principal agente, o Estado, que são: as redistributivas, as distributivas e as regulatórias (AZEVEDO, 2003; LOWI, 1966). A redistributiva consiste em redistribuição de "renda na forma de recursos e/ou de financiamento de equipamentos e serviços públicos" (AZEVEDO, 2003, p. 38). A distributiva representa ações cotidianas de realização compulsória do governo, por meio da oferta de equipamentos e de serviços públicos, de modo pontual ou setorial, considerando a demanda social ou a pressão imposta por grupos (OLIVEIRA, 2010). Essas duas políticas estão no campo de ação do poder executivo.

As políticas públicas regulatórias consistem na elaboração das leis autorizativas das políticas públicas redistributiva ou distributiva, sendo, essencialmente, do campo de atuação do poder legislativo (FREY, 2000). Essas regulações das políticas públicas educacionais, decididas pelo governo, incidem no ambiente escolar no que concerne ao ensino-aprendizagem, afetando a avaliação, a regulação e a supervisão (SILVA JUNIOR et al., 2014), além de gerar diversos sujeitos no campo educativo (SILVA, 2019).

Além de fornecer estrutura educacional nas esferas municipal, estadual e federal, o governo é responsável pela implantação de políticas públicas educacionais que promovam acesso igualitário ao ensino. O ENEM compõe o sistema nacional de avaliação educacional, em conjunto com o Sistema de Avaliação da Educação Básica (SAEB) e o Enade, abrangendo, respectivamente, os níveis de Ensino Médio, Fundamental e Superior, e apresenta à sociedade os resultados como parâmetros da qualidade educacional de políticas públicas educacionais (TRAVITZKI, 2013).

Em 1998, foi instituído o ENEM, com o objetivo de avaliar os conhecimentos e o desenvolvimento de competências e de habilidades do estudante que concluía a Educação Básica e, posteriormente, tornou-se pré-requisito para o ingresso 
no Ensino Superior público (COTTA, 2001) com o objetivo de se constituir em alternativa igualitária e padronizada do acesso às vagas públicas das universidades por meio do desempenho dos alunos em avaliações compostas por áreas do conhecimento.

Desse modo, o exame é pautado por uma matriz de referência de eixos cognitivos, que avalia competências das áreas do conhecimento de: (i) Linguagens, Códigos e suas Tecnologias; (ii) Matemática e suas Tecnologias; (iii) Ciências da Natureza e suas Tecnologias; e (iv) Ciências Humanas e suas Tecnologias. Esses eixos avaliam o domínio da linguagem, a compreensão de fenômenos, o enfrentamento de situações-problema, a construção de argumentação e a elaboração de propostas (INEP, 2013).

Essa matriz de referência é recomendada por se tratar de avaliação de larga escala, com o objetivo de indicar habilidades nas etapas de escolarização, sendo capaz de orientar a elaboração de testes e de provas que favoreçam a construção de escalas de proficiência alcançadas pelo aluno.

Embora o ENEM seja um exame por adesão, e de caráter voluntário, as escolas incentivam os alunos a participarem, pelo fato de o exame ser pré-requisito para o ingresso no Ensino Superior (HAGUETTE; PESSOA; VIDAL, 2016). Para tanto, os autores referem-se ao desempenho educacional mensurado pelo ENEM, como limitado à aprendizagem de conhecimentos suficientes para se obter um bom desempenho na prova.

O desempenho educacional, mensurado pelo ENEM, representa parâmetros comparativos, para uso da sociedade e para demais usuários dessa informação, dos efeitos das aplicações das políticas públicas educacionais, da accountability dos serviços prestados e do nível de qualidade de ensino ofertada pelas estruturas educacionais ofertadas, haja vista que, segundo Haguette, Pessoa e Vidal (2016), "a qualidade da escola pública, mesmo em países ricos, varia segundo a renda das famílias de bairros de uma mesma cidade".

Diante disso, pesquisas como a de Barros et al. (2001) investigaram o desempenho educacional de indivíduos de 11 a 25 anos de idade, residentes nas áreas urbanas das regiões Nordeste e Sudeste, considerando fatores associados à qualidade do serviço ofertado, tais como: a disponibilidade e a qualidade dos serviços educacionais, a atratividade local do mercado de trabalho, os recursos financeiros e não-financeiros do núcleo familiar e o volume de recursos da localidade. Esses contextos foram controlados por características pessoais e geográficas. Como 
achados, dentre outros, tem-se que o desempenho educacional é significativamente positivo em escolas que: (i) o percurso (casa-escola) do estudante é mais curto; (ii) a região seja atrativa ao mercado de trabalho; e (iii) com núcleos familiares mais bem estruturados. Esses resultados evidenciam que a localização da escola afeta de modo diverso o desempenho dos estudantes matriculados.

Soares e Sátyro (2008) analisaram o desempenho dos estudantes de escolas do Ensino Básico fundamental entre 1998 e 2005 e evidenciaram que o porte escolar se mostrou relevante para alcance de resultados superiores. No mesmo ano, Lee estudou o desempenho estudantil de escolas católicas particulares com escolas públicas de Ensino Médio, concluindo que escolas de menor porte tiveram maiores possibilidades de apresentar desempenhos superiores.

Com enfoque nas escolas privadas, Adeodato, Santos Filho e Rodrigues (2014) analisaram o desempenho dos alunos egressos de escolas particulares do Brasil, no ENEM, realizado no ano de 2011. Dentre os resultados, destacou-se que a qualidade das escolas é afetada diretamente pelos aspectos econômico-financeiros dos matriculados, e indiretamente pelo posicionamento geográfico da escola.

Noutra pesquisa comparativa, Haguette, Pessoa e Vidal (2016) investigaram as dez escolas da rede estadual do Ensino Médio do Ceará com maiores e menores desempenhos no ENEM 2011, sendo quatro localizadas no interior e seis na capital, com perfis e com localizações diferentes; apenas uma sendo considerada como rural. Dentre os achados, tem-se que os alunos das escolas com desempenhos superiores apresentaram como fatores motivadores o interesse em ingressar no Ensino Superior e no mercado de trabalho, fomentado pela cultura da localidade. Já os discentes das escolas com desempenhos inferiores, em especial a situada na zona rural, apresentaram desmotivação decorrente da falta de oportunidade presente na localidade, que carece de investimentos públicos para a melhoria da infraestrutura e para a atratividade da localidade.

\section{Delineamento metodológico}

Em atendimento ao seu propósito, a pesquisa pode ser classificada como empírica. Martins e Theóphilo (2009) definem que a pesquisa empírica se dedica à observação e à experimentação através de testes com utilização de medidas quantitativas, como forma de minimizar e/ou eliminar interpretações subjetivas.

$\mathrm{Na}$ abordagem do problema, o estudo classifica-se como quantitativo, tendo em vista a adoção de procedimentos para agrupar e tabular dados, com escopo de 
submeter a testes estatísticos e interpretá-los (MARTINS; THEÓPHILO, 2009) para determinar diferenças de desempenho nos IF do Brasil.

A pesquisa utilizou procedimentos documentais como fontes de dados, informações e de evidências reunidos para serem processados em pesquisas posteriores (MARTINS; THEÓPHILO, 2009). Nesta pesquisa utilizaram-se, como principal fonte da pesquisa documental, os resultados do ENEM, disponíveis na base de dados do INEP.

O estudo é censitário, considerando todos os IF do Brasil que participaram do ENEM no período de 2011 a 2015 e tiveram médias por escola divulgadas pelo INEP. Ressalta-se também que o Governo Federal optou por não divulgar, a partir do ano de 2016, as médias por escola, impossibilitando a coleta de dados após 2015. Tal fato não impede que a presente pesquisa identifique evoluções e tendências do desempenho educacional dos IF do Brasil. A delimitação do espectro populacional é considerada não aleatória e limitada pela disponibilidade dos dados. A composição populacional da pesquisa é formada por 295 campi de todas as regiões da federação, compondo uma amostra final de 954 observações e está detalhada na Tabela 1.

Tabela 1. Quantidade de observações por regiões e estados.

\begin{tabular}{|c|c|c|c|c|c|c|}
\hline \multicolumn{7}{|c|}{ PAINEL A - Centro-Oeste (28 campi) } \\
\hline Estados & 2011 & 2012 & 2013 & 2014 & 2015 & Obs. \\
\hline DF & 1 & 1 & 1 & 1 & 1 & 5 \\
\hline GO & 6 & 3 & 11 & 14 & 14 & 48 \\
\hline MS & 0 & 0 & 0 & 2 & 0 & 2 \\
\hline MT & 6 & 6 & 10 & 11 & 11 & 44 \\
\hline Total & 13 & 10 & 22 & 28 & 26 & 99 \\
\hline \multicolumn{7}{|c|}{ PAINEL B - Nordeste (110 campi) } \\
\hline Estados & 2011 & 2012 & 2013 & 2014 & 2015 & Obs. \\
\hline $\mathrm{AL}$ & 4 & 2 & 9 & 10 & 10 & 35 \\
\hline BA & 11 & 8 & 16 & 23 & 20 & 78 \\
\hline CE & 8 & 2 & 4 & 4 & 3 & 21 \\
\hline MA & 9 & 6 & 13 & 12 & 14 & 54 \\
\hline PB & 4 & 3 & 9 & 10 & 10 & 36 \\
\hline PE & 7 & 3 & 9 & 9 & 11 & 39 \\
\hline $\mathrm{PI}$ & 5 & 3 & 11 & 11 & 11 & 41 \\
\hline RN & 4 & 4 & 11 & 14 & 14 & 47 \\
\hline SE & 2 & 2 & 3 & 3 & 4 & 14 \\
\hline Total & 54 & 33 & 85 & 96 & 97 & 365 \\
\hline
\end{tabular}


Continuação

\begin{tabular}{|c|c|c|c|c|c|c|}
\hline \multicolumn{7}{|c|}{ PAINEL C - Norte (38 campi) } \\
\hline Estados & 2011 & 2012 & 2013 & 2014 & 2015 & Obs. \\
\hline$A C$ & 0 & 0 & 2 & 4 & 3 & 9 \\
\hline AM & 4 & 8 & 10 & 10 & 9 & 41 \\
\hline AP & 0 & 0 & 2 & 2 & 2 & 6 \\
\hline PA & 5 & 1 & 5 & 7 & 5 & 23 \\
\hline RO & 2 & 2 & 6 & 6 & 6 & 22 \\
\hline RR & 2 & 0 & 1 & 2 & 2 & 7 \\
\hline TO & 2 & 1 & 4 & 6 & 6 & 19 \\
\hline Total & 15 & 12 & 30 & 37 & 33 & 127 \\
\hline \multicolumn{7}{|c|}{ PAINEL D - Sudeste (67 campi) } \\
\hline Estados & 2011 & 2012 & 2013 & 2014 & 2015 & Obs. \\
\hline ES & 11 & 2 & 15 & 15 & 15 & 58 \\
\hline MG & 16 & 13 & 22 & 23 & 27 & 101 \\
\hline RJ & 11 & 3 & 7 & 7 & 7 & 35 \\
\hline SP & 2 & 1 & 5 & 7 & 10 & 25 \\
\hline Total & 40 & 19 & 49 & 52 & 59 & 219 \\
\hline
\end{tabular}

PAINEL E - Sul (52 campi)

\begin{tabular}{lcccccc}
\hline Estados & $\mathbf{2 0 1 1}$ & $\mathbf{2 0 1 2}$ & $\mathbf{2 0 1 3}$ & $\mathbf{2 0 1 4}$ & $\mathbf{2 0 1 5}$ & Obs. \\
\hline PR & 2 & 2 & 4 & 8 & 10 & 26 \\
RS & 8 & 5 & 17 & 23 & 20 & 73 \\
SC & 6 & 3 & 8 & 13 & 15 & 45 \\
Total & 16 & 10 & 29 & 44 & 45 & 144 \\
Total Geral & $\mathbf{1 3 8}$ & $\mathbf{8 4}$ & $\mathbf{2 1 5}$ & $\mathbf{2 5 7}$ & $\mathbf{2 6 0}$ & $\mathbf{9 5 4}$ \\
\hline
\end{tabular}

DF: Distrito Federal; GO: Goiás; MS: Mato Grosso do Sul; MT: Mato Grosso; AL: Alagoas; BA: Bahia; CE: Ceará; MA: Maranhão; PB: Paraíba; PE: Pernambuco; Pl: Piauí; RN: Rio Grande do Norte; SE: Sergipe; AC: Acre; AM: Amazonas; AP: Amapá; PA: Pará; RO: Roraima; RR: Rondônia; TO: Tocantins; ES: Espírito Santo; MG: Minas Gerais; RJ: Rio de Janeiro; SP: São Paulo; PR: Paraná; RS: Rio Grande do Sul; SC: Santa Catarina. Fonte: Elaborada pelos autores (2019).

Para o teste de hipótese, consideraram-se os desempenhos médios dos Institutos Federais de Educação, Ciência e Tecnologia do Brasil nas respectivas áreas de conhecimento e média geral do ENEM por instituição, definidos e descritos na operacionalização (Quadro). 
Quadro1. Definição e operacionalização das variáveis e categorias de análise*.

\begin{tabular}{|c|c|c|c|}
\hline Variável & & Operacionalização & Fonte \\
\hline \multirow[t]{2}{*}{ Linguagens e Códigos } & \multirow{2}{*}{$\mathrm{LC}=$} & $\Sigma$ Notas de Linguagens e Códigos & \multirow[t]{2}{*}{ INEP } \\
\hline & & Total de alunos & \\
\hline \multirow{2}{*}{$\begin{array}{l}\text { Matemática e suas } \\
\text { Tecnologias }\end{array}$} & \multirow{2}{*}{$\mathrm{MT}=$} & $\Sigma$ Notas de Matemática e suas Tecnologias & \multirow[t]{2}{*}{ INEP } \\
\hline & & Total de alunos & \\
\hline \multirow[t]{2}{*}{ Ciências Humanas } & \multirow{2}{*}{$\mathrm{CH}=$} & $\Sigma$ Notas de Ciências Humanas & \multirow[t]{2}{*}{ INEP } \\
\hline & & Total de alunos & \\
\hline \multirow[t]{2}{*}{ Ciências da Natureza } & \multirow{2}{*}{$\mathrm{CH}=$} & $\Sigma$ Notas de Ciências da Natureza & \multirow[t]{2}{*}{ INEP } \\
\hline & & Total de alunos & \\
\hline \multirow[t]{2}{*}{ Redação } & \multirow{2}{*}{$\mathrm{RED}=$} & $\Sigma$ Notas de Redação & \multirow[t]{2}{*}{ INEP } \\
\hline & & Total de alunos & \\
\hline \multirow[t]{2}{*}{ Nota ENEM } & \multirow{2}{*}{ ENEM $=$} & $\Sigma$ Médias do ENEM & \multirow[t]{2}{*}{ INEP } \\
\hline & & Total de alunos & \\
\hline
\end{tabular}

INEP: Instituto Nacional de Estudos e Pesquisas Educacionais Anísio Teixeira; ENEM: Exame Nacional do Ensino Médio.

* As notas do ENEM, e de cada componente, representam uma média da nota de todos os alunos participantes por instituição.

Fonte: Elaborado pelos autores (2019).

Em atendimento ao objetivo da pesquisa - investigar os níveis diferenciados da proficiência obtida pelos IF do Brasil no ENEM - e à hipótese do estudo - o desempenho alcançado pelas instituições federais de Ensino Básico, no transcorrer do período, é crescente e significante - a seção de análise de resultados foi dividida em duas partes: análise descritiva e comparativa do desempenho dos IF no ENEM.

A análise descritiva, de correlação e comparativa da evolução do desempenho no ENEM foi realizada por região, por zona e por porte da instituição. Sendo assim, a amostra foi subdividida nas categorias anteriormente citadas. Foi utilizada a análise de variância (Anacor) para comparar o desempenho no ENEM no período analisado.

\section{Análise de resultados e discussão}

Esta seção destina-se a apresentar os resultados da análise descritiva da amostra da pesquisa, assim como o desempenho dos IF do Brasil, comparativamente à região, à zona e ao tamanho institucional. Inicialmente apresenta-se, na Tabela 2, a média, o desvio-padrão e os coeficientes de correlação das notas do ENEM, divulgadas pelos 295 campi. 
Tabela 2. Análise descritiva e de correlação.

\begin{tabular}{|c|c|c|c|c|c|c|c|c|}
\hline Variável & Média & DP & LC & MT & $\mathrm{CH}$ & $\mathrm{CN}$ & RED & ENEM \\
\hline LC & 541,50 & 32,94 & 1,00 & - & - & - & - & - \\
\hline MT & 560,67 & 64,77 & $0,77^{* \star *}$ & 1.00 & - & - & - & - \\
\hline $\mathrm{CH}$ & 575,93 & 40,39 & $0,68^{* \star *}$ & $0,42^{* * *}$ & 1.00 & - & - & - \\
\hline $\mathrm{CN}$ & 522,25 & 36,46 & $0,86^{* \star *}$ & $0,81^{\star \star \star}$ & $0,75^{\star \star \star}$ & 1,00 & - & - \\
\hline RED & 591,59 & 54,27 & $0,77^{\star \star \star}$ & $0,61^{* * *}$ & $0,68^{* * *}$ & $0,71^{\star * *}$ & 1,00 & - \\
\hline ENEM & 557,23 & 39,77 & $0,90^{* \star *}$ & $0,84^{\star \star \star}$ & $0,81^{* * *}$ & $0,94^{* * *}$ & $0,85^{\star \star *}$ & 1,00 \\
\hline
\end{tabular}

LC: Linguagens e Códigos; MT: Matemática e suas Tecnologias; $\mathrm{CH}$ : Ciências Humanas; CN: Ciências da Natureza; RED: Redação; ENEM: Média Geral do Exame Nacional do Ensino Médio. *** Representa o nível de significância de $1 \%$.

Fonte: Elaborada pelos autores (2019).

De modo geral, observa-se que a maior média ocorreu no componente de Redação, seguida pelo componente de Ciências Humanas, o que evidencia a presença de discentes com habilidades e competências despontadas nessas áreas, contribuindo com a média final no referido exame das Instituições Federais em 557,23 pontos, considerando o conjunto populacional estudado. Com relação à análise de correlação, é possível perceber que o desempenho de todos os componentes da avaliação no ENEM está positivamente correlacionado, não sendo observadas discrepâncias; e isso conserva o mesmo sentido em todas as áreas avaliadas, o que denota sinergia entre elas, considerando os resultados dos estudantes. A variabilidade entre as médias é considerada pequena, indicando baixa dispersão entre elas, o que demonstra que os IF apresentam resultados similares, com ausência de outliers evidentes, presença de política institucional e educacional equalizada para seus diferentes públicos das diversas localidades do Brasil.

Apresenta-se, na Tabela 3, a comparação das médias de desempenho do ENEM, ao longo do período analisado, dos IF que participaram do exame. Em todos os componentes, as médias de desempenho se mostraram significativamente diferentes.

Tabela 3. Análise comparativa dos Institutos da Rede de Ensino Federal.

\begin{tabular}{|c|c|c|c|c|c|c|}
\hline Período & LC & MT & $\mathrm{CH}$ & $\mathrm{CN}$ & RED & ENEM \\
\hline 2011 & 559,53 & 603,63 & 526,81 & 521,69 & 592,99 & 552,91 \\
\hline 2012 & 571,04 & 568,54 & 557,17 & 512,85 & 567,06 & 544,53 \\
\hline 2013 & 529,05 & 575,68 & 564,73 & 511,91 & 583,32 & 552,94 \\
\hline 2014 & 549,25 & 550,43 & 594,44 & 536,32 & 589,75 & 564,04 \\
\hline 2015 & 542,46 & 533,05 & 599,04 & 520,21 & 607,42 & 560,43 \\
\hline Teste F & $38,44^{\star * *}$ & $36,52^{* \star *}$ & $155,87^{\star \star * *}$ & $16,51^{\star \star *}$ & $11,67^{\text {*** }}$ & $5,59 * * *$ \\
\hline Estatística & 0,00 & 0,00 & 0,00 & 0,00 & 0,00 & 0,00 \\
\hline
\end{tabular}

LC: Linguagens e Códigos; MT: Matemática e suas Tecnologias; $\mathrm{CH}$ : Ciências Humanas; CN: Ciências da Natureza; RED: Redação; ENEM: Média Geral do Exame Nacional do Ensino Médio. *** Representa o nível de significância de $1 \%$.

Fonte: Elaborada pelos autores (2019). 
Analisando, inicialmente, o resultado final, nota no ENEM, observa-se que há uma evolução crescente de desempenho dos alunos das Instituições Federais de ensino, contudo, a evolução apresenta crescimento moderado. Os dados apontam, de modo geral, que as áreas do conhecimento de Ciências Humanas e de Redação apresentaram médias crescentes entre os períodos, evidenciando que seus discentes vêm apresentando níveis de apreensão de conhecimento, habilidades e conhecimentos superiores para as áreas de conhecimento listadas. $\mathrm{O}$ mesmo não foi detectado com consistência nas médias das áreas de Linguagens e Códigos e de Ciências da Natureza, que oscilaram entre os períodos, não estabelecendo um padrão para crescimento ou decrescimento consistentes. Já a área de Matemática e suas Tecnologias demonstrou redução das médias a cada período, porém, apesar do declínio, é perceptível que as diferenças não são elásticas, reduzindo a cada período. Esses resultados contribuíram para que a média do ENEM oscilasse entre os períodos, estabelecendo padrão visível de tendência ao crescimento moderado.

Apesar de os resultados encontrados não apresentarem unanimidade de crescimento positivo e significativo em todas as áreas, opta-se por confirmar a hipótese de pesquisa considerando que os desempenhos nas áreas de conhecimento impactaram positiva e significantemente a média do ENEM de todo o conjunto amostral.

Posteriormente, analisa-se a evolução do desempenho dos educandos no ENEM, com destaque à região, buscando complementar a análise do estudo. A Tabela 4 evidencia os resultados dos testes de comparação de médias por região.

Tabela 4. Testes de comparação entre médias por região.

\begin{tabular}{|c|c|c|c|c|c|c|}
\hline \multicolumn{7}{|c|}{ PAINEL A - Região Norte } \\
\hline Período & LC & MT & $\mathrm{CH}$ & $\mathrm{CN}$ & RED & ENEM \\
\hline 2011 & 553,99 & 588,24 & 525,12 & 513,23 & 576,68 & 545,15 \\
\hline 2012 & 508,35 & 550,36 & 548,94 & 507,85 & 548,75 & 532,85 \\
\hline 2013 & 529,59 & 569,17 & 565,34 & 514,62 & 577,07 & 551,16 \\
\hline 2014 & 543,80 & 540,84 & 592,44 & 539,18 & 583,50 & 559,95 \\
\hline 2015 & 539,89 & 525,87 & 598,48 & 522,20 & 602,41 & 557,77 \\
\hline Teste F & $7,44^{\star * *}$ & 4,44 & $24,65^{* * *}$ & $3,20^{* *}$ & $4,01^{* * *}$ & 1,49 \\
\hline Estatística & 0,000 & 0,002 & 0,000 & 0,016 & 0,004 & 0,210 \\
\hline \multicolumn{7}{|c|}{ PAINEL B - Região Nordeste } \\
\hline Período & LC & MT & $\mathrm{CH}$ & $\mathrm{CN}$ & RED & ENEM \\
\hline 2011 & 552,74 & 574,97 & 517,21 & 505,32 & 595,39 & 537,56 \\
\hline 2012 & 515,36 & 554,67 & 555,25 & 505,15 & 566,59 & 539,41 \\
\hline 2013 & 519,28 & 554,22 & 555,41 & 495,38 & 579,38 & 540,73 \\
\hline 2014 & 542,34 & 528,20 & 586,32 & 524,39 & 584,22 & 553,09 \\
\hline 2015 & 530,84 & 504,35 & 590,41 & 504,91 & 603,07 & 546,71 \\
\hline Teste F & $17,80^{* * *}$ & $22,55^{* * *}$ & $71,42^{* * *}$ & $12,79 * * *$ & $3,87^{* * *}$ & $2,40^{\star *}$ \\
\hline Estatística & 0,000 & 0,000 & 0,000 & 0,000 & 0,004 & 0,049 \\
\hline
\end{tabular}


Continuação

\begin{tabular}{|c|c|c|c|c|c|c|}
\hline \multicolumn{7}{|c|}{ PAINEL C - Região Centro-Oeste } \\
\hline Período & LC & MT & $\mathrm{CH}$ & $\mathrm{CN}$ & RED & ENEM \\
\hline 2011 & 553,99 & 588,24 & 525,12 & 513,23 & 576,68 & 545,15 \\
\hline 2012 & 508,35 & 550,36 & 548,94 & 507,85 & 548,75 & 532,85 \\
\hline 2013 & 529,59 & 569,17 & 565,34 & 514,62 & 577,07 & 551,16 \\
\hline 2014 & 543,80 & 540,84 & 592,44 & 539,18 & 583,50 & 559,95 \\
\hline 2015 & 539,89 & 525,87 & 598,48 & 522,20 & 602,41 & 557,77 \\
\hline Teste F & $5,73^{\star * *}$ & $8,07^{\star * *}$ & $26,06^{\star * *}$ & $5,97^{\star \star *}$ & $2,77^{\star *}$ & $2,19 *$ \\
\hline Estatística & 0,000 & 0,000 & 0,000 & 0,000 & 0,032 & 0,076 \\
\hline \multicolumn{7}{|c|}{ PAINEL D - Região Sudeste } \\
\hline Período & LC & MT & $\mathrm{CH}$ & $\mathrm{CN}$ & RED & ENEM \\
\hline 2011 & 577,02 & 655,32 & 546,54 & 552,32 & 605,49 & 582,79 \\
\hline 2012 & 534,46 & 608,97 & 575,32 & 535,54 & 594,54 & 569,77 \\
\hline 2013 & 553,92 & 631,32 & 593,23 & 547,86 & 612,45 & 587,76 \\
\hline 2014 & 568,85 & 606,32 & 616,84 & 568,05 & 630,87 & 598,19 \\
\hline 2015 & 561,61 & 587,56 & 615,14 & 548,51 & 629,84 & 588,53 \\
\hline Teste F & $9,53^{\star \star *}$ & 10,69 *** & $46,44^{\star \star \star}$ & $4,54^{\star \star \star}$ & $3,94^{* * *}$ & $2,57^{\star *}$ \\
\hline Estatística & 0,000 & 0,000 & 0,000 & 0,002 & 0,004 & 0,039 \\
\hline \multicolumn{7}{|c|}{ PAINEL E - Região Sul } \\
\hline Período & LC & MT & $\mathrm{CH}$ & $\mathrm{CN}$ & RED & ENEM \\
\hline 2011 & 566,71 & 634,46 & 535,55 & 536,75 & 591,16 & 568,38 \\
\hline 2012 & 530,95 & 596,96 & 570,11 & 536,25 & 565,42 & 559,94 \\
\hline 2013 & 538,95 & 594,16 & 569,32 & 524,44 & 578,18 & 561,01 \\
\hline 2014 & 561,53 & 578,13 & 604,83 & 546,04 & 593,28 & 576,76 \\
\hline 2015 & 556,43 & 556,47 & 608,52 & 528,99 & 606,44 & 571,37 \\
\hline Teste F & $7,14^{\star \star *}$ & $10,69 * * *$ & $37,81^{\star \star \star}$ & $3,70^{\star \star \star}$ & $2,36^{*}$ & 1,52 \\
\hline Estatística & 0,000 & 0,000 & 0,000 & 0,007 & 0,056 & 0,198 \\
\hline
\end{tabular}

LC: Linguagens e Códigos; MT: Matemática e suas Tecnologias; CH: Ciências Humanas; CN: Ciências da Natureza; RED: Redação; ENEM: Média Geral do Exame Nacional do Ensino Médio. *; **; *** Representa o nível de significância de 10\%, 5\% e 1\%, respectivamente.

Fonte: Elaborada pelos autores (2019).

A comparação de médias por região mostra diferenças significativas em todos os componentes, contudo, no desempenho final, medido pela nota no ENEM, não foi significativa nas regiões Norte e Sul. Além disso, apesar da proximidade das médias, foram detectadas diferenças entre as proficiências auferidas entre regiões, corroborando com as pesquisas de Barros et al. (2001) e de Adeodato, Santos Filho e Rodrigues (2014), que atribuem essas diferenças a características pessoais existentes em cada localidade. De modo geral, aqui também se opta por confirmar a hipótese de pesquisa de crescimento positivo e significante entre os períodos, considerando a segmentação por regiões. 
Em outro momento, analisa-se a região na qual as instituições estão localizadas - zona urbana ou rural. A Tabela 5 apresenta os testes de comparação de média por zona.

Tabela 5. Testes de comparação entre médias por zona.

\begin{tabular}{|c|c|c|c|c|c|c|}
\hline \multicolumn{7}{|c|}{ PAINEL A - Zona Rural } \\
\hline Período & LC & MT & $\mathrm{CH}$ & $\mathrm{CN}$ & RED & ENEM \\
\hline 2011 & 535,66 & 568,91 & 505,76 & 500,64 & 555,65 & 527,74 \\
\hline 2012 & 509,08 & 556,85 & 548,41 & 506,96 & 544,57 & 533,18 \\
\hline 2013 & 512,84 & 561,11 & 549,79 & 504,20 & 552,23 & 536,03 \\
\hline 2014 & 535,35 & 534,29 & 580,46 & 527,93 & 559,39 & 547,48 \\
\hline 2015 & 527,31 & 513,04 & 586,98 & 508,88 & 586,11 & 544,46 \\
\hline Teste & $9,51^{\star \star \star}$ & $10,55^{\star \star \star}$ & $63,11^{\star * *}$ & $5,94^{\star * *}$ & $4,81^{\star \star \star}$ & $2,56^{\star *}$ \\
\hline Estatística & 0,000 & 0,000 & 0,000 & 0,000 & 0,001 & 0,039 \\
\hline \multicolumn{7}{|c|}{ PAINEL B - Zona Urbana } \\
\hline Período & LC & MT & $\mathrm{CH}$ & $\mathrm{CN}$ & RED & ENEM \\
\hline 2011 & 541,75 & 560,30 & 579,00 & 523,19 & 593,16 & 558,52 \\
\hline 2012 & 542,73 & 560,81 & 576,98 & 522,83 & 593,38 & 558,14 \\
\hline 2013 & 543,05 & 560,65 & 577,35 & 523,22 & 593,72 & 558,37 \\
\hline 2014 & 541,84 & 562,13 & 575,68 & 521,93 & 593,37 & 557,77 \\
\hline 2015 & 542,35 & 563,53 & 575,27 & 523,05 & 591,92 & 557,99 \\
\hline Teste F & $31,03^{\star * *}$ & $31,40^{* \star *}$ & $100,04^{\star \star \star}$ & $11,93^{* \star *}$ & $6,38^{\star \star \star}$ & $2,47^{\star *}$ \\
\hline Estatística & 0,000 & 0,000 & 0,000 & 0,000 & 0,000 & 0,043 \\
\hline
\end{tabular}

LC: Linguagens e Códigos; MT: Matemática e suas Tecnologias; CH: Ciências Humanas; CN: Ciências da Natureza; RED: Redação; ENEM: Média Geral do Exame Nacional do Ensino Médio. ***; ** Significância estatística ao nível de $1 \%$ e $5 \%$, respectivamente.

Fonte: Elaborado pelos autores (2019).

Os resultados mostraram-se significativos em todos os componentes analisados. Como destaque, apesar de, em termos absolutos, as médias dos IF da zona urbana serem superiores às da zona rural, tem-se o desempenho desses últimos, consistentemente positivos e significantes nas áreas de conhecimento de Ciências Humanas e Redação, entre os anos estudados.

Por outro lado, os IF localizados na zona urbana demonstraram desenvoltura positiva e significativa na área de Matemática e suas Tecnologias, apesar do baixo coeficiente explicativo, não sendo observado nas unidades situadas na zona rural. Tal resultado não corroborou com a pesquisa de Haguette, Pessoa e Vidal (2016), que detectou em escola rural menores desempenhos. 
Vale lembrar que o período estudado coincide com o processo de expansão e de interiorização dos Institutos Federais, e que essas informações podem auxiliar e direcionar a implantação de cursos mais bem adequados aos anseios da localidade.

Assim, apesar do crescimento, para o comparativo por zona demográfica, opta-se por rejeitar a hipótese de pesquisa, pois, diferentemente da segmentação regional, as unidades estão sujeitas, por exemplo, a comparações com outras de contextos diferentes, e, portanto, os resultados, quando analisados por essa segmentação, impactam de modo significante a média final no transcorrer dos anos, mas com baixo coeficiente explanatório.

Por fim, analisa-se a evolução do desempenho, considerando o porte de cada unidade. $\mathrm{O}$ agrupamento foi feito pelo quantitativo de estudantes na instituição. A Tabela 6 apresenta os testes de comparação de média por faixa (porte).

Tabela 6. Testes de comparação entre médias por porte da instituição.

\begin{tabular}{lcccccc}
\hline \multicolumn{7}{c}{ PAINEL A - De 0 a 90 alunos } \\
\hline Período & LC & MT & CH & CN & RED & ENEM \\
2011 & 538,54 & 553,94 & 583,06 & 522,09 & 590,95 & 557,62 \\
2012 & 543,15 & 559,74 & 577,14 & 522,77 & 593,31 & 557,97 \\
2013 & 543,60 & 557,25 & 577,78 & 524,03 & 592,82 & 557,71 \\
2014 & 539,44 & 561,75 & 571,53 & 518,71 & 591,10 & 555,10 \\
2015 & 541,36 & 566,27 & 571,17 & 522,83 & 588,67 & 556,66 \\
Teste F & $9,77^{* * *}$ & $5,79 * *$ & $40,72^{* * *}$ & $5,43^{* * *}$ & $5,38^{* * *}$ & $3,15^{* *}$ \\
Estatística & 0,000 & 0,000 & 0,000 & 0,000 & 0,000 & 0,015 \\
\hline \multicolumn{7}{c}{ PAINEL B - Maior que 90 alunos } \\
\hline Período & LC & MT & CH & CN & RED & ENEM \\
2011 & 561,25 & 605,77 & 528,21 & 523,23 & 595,85 & 554,61 \\
2012 & 519,59 & 572,99 & 560,00 & 515,33 & 568,89 & 547,36 \\
2013 & 530,46 & 578,57 & 566,29 & 512,91 & 585,15 & 554,68 \\
2014 & 550,90 & 555,72 & 596,08 & 538,42 & 593,80 & 566,99 \\
2015 & 542,12 & 534,27 & 598,39 & 519,47 & 605,35 & 559,92 \\
Teste F & $29,54 * * *$ & $27,26 * * *$ & $115,28^{* * *}$ & $12,42 * * *$ & $7,50 * * *$ & $4,02 * * *$ \\
Estatística & 0,000 & 0,000 & 0,000 & 0,000 & 0,000 & 0,003 \\
\hline
\end{tabular}

LC: Linguagens e Códigos; MT: Matemática e suas Tecnologias; CH: Ciências Humanas; CN: Ciências da Natureza; RED: Redação; ENEM: Média Geral do Exame Nacional do Ensino Médio. *** Significância estatística ao nível de $1 \%$.

Fonte: Elaborado pelos autores (2019). 
Para essa análise, as evidências sugerem que o desempenho educacional nas instituições com mais de 90 estudantes matriculados é positivo e significante em Ciências Humanas e em Redação, enquanto que as de menor porte, em Matemática e suas Tecnologias, no decorrer dos períodos. Os resultados divergem de Soares e Sátyro (2008) e de Lee (2008), não mostrando ser contexto suficiente para impor superioridade de desempenho. Apesar de os resultados nas áreas do conhecimento serem estatisticamente relevantes, rejeita-se a hipótese de pesquisa por considerar o baixo poder explicativo do coeficiente.

Assim, na presente pesquisa, conclui-se, de modo geral, que os resultados aferidos pelos Institutos Federais do Brasil, quando analisados como Rede de Ensino e por localização regional, apresentam evolução positiva e significante no transcorrer dos anos, confirmando a hipótese levantada, e apontando indícios de que, apesar das diferenças sociais e culturais das regiões, esses fatores não afetam o rendimento crescente da rede de ensino, pois cada unidade evolui em conformidade com o contexto em que se encontra inserida. O mesmo não ocorre na análise por zona demográfica e por porte escolar.

\section{Considerações finais}

Em observância à Teoria da Função de Produção Educacional, para aprimorar o emprego de insumos, o presente artigo teve como objetivo investigar os níveis diferenciados da proficiência obtida pelos IF do Brasil no ENEM. Para a elucidação do proposto e verificação da hipótese de pesquisa, utilizaram-se os resultados do ENEM, por escola, divulgados pelo INEP, nos períodos de 2011 a 2015.

Os principais resultados verificados pela análise descritiva de dados evidenciaram que os desempenhos das médias nas áreas de Ciências Humanas e de Redação mostraram-se crescentes nos transcorrer dos anos estudados; para Linguagens e Códigos e Ciências da Natureza apresentaram acréscimos de performances a partir de 2013; por outro lado, Matemática e suas Tecnologias obtiveram médias decrescentes em todos os períodos. Esses resultados compuseram a média geral do ENEM, que apresentou rendimentos crescentes e significativos para análise da Rede de Ensino Profissional. Além disso, esses resultados revelaram que as notas dos IF do Brasil no ENEM apresentam proximidade com a média e, por consequência, ausência de notas extremas (outliers).

Da mesma maneira, as médias, quando analisadas em cada região, evidenciaram proficiências positivas e significativas respeitando os estágios e as estratégias educacionais para a implantação das políticas públicas na Rede Federal de Ensino Básico, harmônicas com a realidade local. A coerência da análise é visualizada no 
momento em que os desempenhos são confrontados com fatores gerais, como o contexto zonal e de porte escolar. Nesses, a comparação não é realizada em forma de painel que agrega características (contextos) semelhantes, como, por exemplo, tempo de funcionamento, perfil socioeconômico discente, potencialidade da região e estágio do ciclo de vida, não sendo razoável gerar expectativas similares em condições diversas.

Ressalta-se que a pesquisa teve como limitações a ausência de publicações de dados relativos às médias por escola dos anos posteriores a 2015, por decisão do Governo Federal. Porém, tais restrições não impediram as inferências estatísticas para determinar a evolução e as tendências do desempenho educacional dos IF do Brasil.

É possível sugerir, para pesquisas futuras, o estudo empírico comparativo com uso de métricas provenientes do censo do Índice de Desenvolvimento da Educação Básica (IDEB), considerando contextos como tempo de funcionamento, perfil socioeconômico discente, potencialidade da região e estágio do ciclo de vida, aos quais as instituições de ensino estejam inseridas. 


\title{
What has changed in the educational performance of Federal Institutes of Brazil?
}

\begin{abstract}
The research investigated the different levels of proficiency obtained by the Federal Institutes of Brazil in the ENEM, since it is understood that the public policies adopted nationally for the Federal Network of Education strengthen over time the offer of education. In order to meet the proposed objective, the studied population was composed of all the Federal Institutes of Brazil that disseminated data regarding the educational proficiency obtained by means of the ENEM knowledge areas, per school, extracted from the INEP electronic site in the period from 2011 to 2015 The methodological course was established by univariate statistical methods of measures of central tendency, dispersion, correlation and comparison of means between variables. Based on the findings, it was verified that the performance of the Federal Institutes of Brazil tends to be increasing and significant over the years, both when evaluated as a teaching network and when evaluated by region.
\end{abstract}

Keywords: Production function theory. Educational performance. Public educational policies. ENEM.

\section{¿Qué cambió en el desempeño educativo de los Institutos Federales de Brasil?}

\section{Resumen}

La investigación investigó los niveles diferenciados de la competencia obtenida por los Institutos Federales de Brasil en el ENEM, pues se comprende que las políticas públicas adoptadas nacionalmente para la Red Federal de Enseñanza fortalecen a lo largo del tiempo la oferta de enseñanza. Para atender el objetivo propuesto, la población estudiada fue compuesta por todos los Institutos Federales de Brasil que divulgar datos referentes a la proficiencia educacional obtenida por las medias de las áreas del conocimiento del ENEM, por escuela, extraídos del sitio electrónico del INEP en el período de 2011 a 2015 El recorrido metodológico fue establecido por métodos estadísticos univariados de medidas de tendencia central, de dispersión, de correlación y de comparación de promedios entre las variables. Con base en los hallazgos, se verificó que el desempeño de los Institutos Federales de Brasil tiende a ser creciente y significante en el transcurso de los años, tanto cuando se evalúa como red de enseñanza, cuanto cuando es evaluado por región.

Palabras clave: Teoría de la función de producción. Desempeño educativo. Políticas públicas educativas. ENEM. 


\section{Referências}

ADEODATO, P. J. L.; SANTOS FILHO, M. M.; RODRIGUES, R. L. Predição de desempenho de escolas privadas usando o Enem como indicador de qualidade escolar. In: SIMPÓSIO BRASILEIRO DE INFORMÁTICA NA EDUCAÇÃO, 25. 2014, Dourados. Anais... Dourados, GO: Sociedade Brasileira de Computação, 2014.

ALMEIDA, N. C. Análise dos determinantes da proficiência dos cursos de engenharia no Enade 2011 e 2014. 2018. 39 f. Dissertação (Mestrado em economia) - Universidade Federal do Ceará, Faculdade de Economia, Administração, Atuariais, Contabilidade e Secretariado Executivo, Fortaleza, CE, 2018.

AZEVEDO, S. Políticas públicas: discutindo modelos e alguns problemas de implementação. In. SANTOS JUNIOR, A. O. et al. (Orgs.). Políticas públicas e gestão local: programa interdisciplinar de capacitação de conselheiros municipais. Rio de Janeiro, RJ: Fase, 2003. p. 38-44.

BARROS, R. P. D. et al. Determinantes do desempenho educacional no Brasil. Pesquisa Planejamento \& Economia, Brasília, v. 31, n. 1, p. 1-12, abr. 2001.

BECHER, E. L.; TOMASI, F. M. A.; JUSTO, J. C. R. Provinha e prova Brasil de matemática nas práticas pedagógicas dos professores de matemática. Pesquisa em Foco, São Luís, v. 23, n. 1, p. 59-81, jan./jun. 2018. https://doi.org/10.18817/pef.v23i1.1653

BELTRÃO, K. I.; MANDARINO, M. C. F. Evidências do Enade: mudanças no perfil do matemático graduado. Ensaio: Avaliação e Políticas Públicas em Educação, Rio de Janeiro, v. 22, n. 84, p. 733-54, jul./set. 2014. https://doi.org/10.1590/S0104-40362014000300007

BOWLES, S. Towards and educational production function. In: HANSEN, W. L. (Ed.). Education, income, and human capital. New York, NY: National Bureau of Economic Research, 1970. p. 9-70. Disponível em: $<$ http://www. nber.org/>. Acesso em: 9 nov. 2017.

COLEMAN, J. S. et al. Equality of educational opportunity. Washington, DC: National Center for Educational Statistics, 1966.

COTTA, T. C. Avaliação educacional e políticas públicas: a experiência do sistema nacional de avaliação da educação básica (Saeb). Revista do Serviço Público, v. 52, n. 4, p. 89-111, out./dez., 2001. https://doi.org/10.21874/rsp.v52i4.316 
FREY, K. Políticas públicas: um debate conceitual e reflexões referentes à prática da análise de políticas públicas no Brasil. Planejamento e Políticas Públicas, Brasília, n. 21, p. 211-59, 2000.

HAGUETTE, A.; PESSOA, M. K. M.; VIDAL, E. M. Dez escolas, dois padrões de qualidade: uma pesquisa em dez escolas públicas de ensino médio do estado do Ceará. Ensaio: Avaliação e Políticas Públicas em Educação, Rio de Janeiro, v. 24, n. 92, p. 609-36, jun./set. 2016. https://doi.org/10.1590/S0104-40362016000300005

INSTITUTO NACIONAL DE ESTUDOS E PESQUISAS EDUCACIONAIS ANÍSIO TEIXEIRA - INEP. Matriz de referência. Brasília, DF: Ministério da Educação, 2013. Disponível em: <http://portal.inep.gov.br/web/guest/matrizde-referencia>. Acesso em: 6 jan. 2018.

INSTITUTO NACIONAL DE ESTUDOS E PESQUISAS EDUCACIONAIS ANÍSIO TEIXEIRA - INEP. Microdados. Brasília, DF: Ministério da Educação, 2015. Disponível em: <http://portal.inep.gov.br/web/guest/ microdados>. Acesso em: 9 nov. 2017.

INSTITUTO NACIONAL DE ESTUDOS E PESQUISAS EDUCACIONAIS ANÍSIO TEIXEIRA - INEP. Brasil no PISA 2015: análises e reflexões sobre o desempenho dos estudantes brasileiros. Brasília, DF: Ministério da Educação, 2016.

INSTITUTO NACIONAL DE ESTUDOS E PESQUISAS EDUCACIONAIS ANÍSIO TEIXEIRA - INEP. Censo escolar da educação básica 2016: notas estatísticas. Brasília, DF: Ministério da Educação, 2017. Disponível em: $<$ http://download.inep.gov.br/educacao_basica/censo_escolar/notas estatisticas/2017/notas_estatisticas_censo_escolar_da_educacao_basica_2016. pdf>. Acesso em: 9 nov. 2017.

LEE, V. Utilização e modelos hierárquicos lineares para estudar contextos sociais. In: BROOKE, N.; SOARES, J. F. (Eds.). Pesquisa em eficácia escolar: origem e trajetórias. Belo Horizonte, MG: Universidade Federal de Minas Gerais, 2008. p. 273-98

LOWI, T. J. Distribution, regulation, redistribution: the functions of government. In: RIPLEY, R. B. (Ed.). Public policies and their politics: techniques of government control. New York, NY: WW Norton, 1966. p. 27-40

LUZ, L. S. Os determinantes do desempenho escolar: a estratificação educacional e o efeito valor adicionado. In: ENCONTRO NACIONAL DE 
ESTUDOS POPULACIONAIS, 15. 2006, Caxambú. Anais... Caxambú, MG: Associação Brasileira de Estudos Populacionais, 2006.

MARTINS, G. A.; THÉOPHILO, C. R. Metodologia da investigação científica para ciências sociais aplicadas. São Paulo, SP: Atlas, 2009.

MENALDO, B. E. A utilização do Enade como métrica de qualidade dos cursos de ensino superior. 2018. 56 f. Dissertação (Mestrado profissional em finanças e economia) - Fundação Getúlio Vargas, Escola de Economia de São Paulo, São Paulo, SP, 2018.

OLIVEIRA, A. F. Políticas públicas educacionais: conceito e contextualização numa perspectiva didática. In: OLIVEIRA, A. F.; PIZZIO, A.; FRANÇA, G. Fronteiras da educação: desigualdades, tecnologias e políticas. Goiania, GO: Pontifícia Universidade Católica de Goiás, 2010. p. 93-9

PAIVA, P. B.; FREIRE, F. S.; FERNANDES, J. L. T. Avaliando o curso de ciências contábeis: uma visão dos alunos da UnB. Ensaio: Avaliação e Políticas Públicas em Educação, Rio de Janeiro, v. 20, n. 74, p. 89-113, jan./ mar. 2012. https://doi.org/10.1590/S0104-40362012000100006

RAMOS, M. N. Ensino médio na rede federal e nas redes estaduais: por que os estudantes alcançam resultados diferentes nas avaliações de larga escala? Holos, Rio Grande do Norte, v. 2, p. 449-59, 2018. https://doi.org/10.15628/ holos.2018.6976

ROSISTOLATO, R.; PRADO, A. P.; MARTINS, L. R. A "realidade" de cada escola e a recepção de políticas educacionais. Ensaio: Avaliação e Políticas Públicas em Educação, Rio de Janeiro, v. 26, n. 98, p. 112-32, jan./mar. 2018. https://doi.org/10.1590/s0104-40362018002601074

SALGADO JUNIOR, A. P.; NOVI, J. C. Proposta de práticas administrativo-pedagógicas que possam contribuir para o desempenho dos alunos de escolas municipais do ensino fundamental na prova Brasil. Ensaio: Avaliação e Políticas Públicas em Educação, Rio de Janeiro, v. 23, n. 88, p. 631-62, jul./set. 2015. https://doi.org/10.1590/S0104-40362015000300005

SILVA, R. R. D. A individualização dos percursos formativos como princípio organizador das políticas curriculares para o ensino médio no Brasil. Ensaio: Avaliação e Políticas Públicas em Educação, Rio de Janeiro, v. 27, n. 103, p. 426-47, abr./jun. 2019. https://doi.org/10.1590/s0104-40362018002601254 
SILVA JUNIOR, A. et al. Políticas públicas para a educação superior: a avaliação, a regulação e a supervisão de IES privadas em debate. Ensaio: Avaliação e Políticas Públicas em Educação, Rio de Janeiro, v. 22, n. 82, p. 215-40, jan./mar. 2014. https://doi.org/10.1590/S0104-40362014000100011

SLONIAK, M. A. et al. Politica pública de educação uma análise do exame nacional do ensino médio (Enem) no Distrito Federal. Universitas/Jus, Brasília, v. 24, n. 1, p. 61-78, jan./jun. 2013. https://doi.org/10.5102/unijus. v24i1.2259

SOARES, S.; SÁTYRO, N. O impacto de infra-estrutura escolar na taxa de distorção idade-série das escolas brasileiras de ensino fundamental: 1998 a 2005. Brasília, DF: Instituto de Pesquisa Econômica Aplicada, 2008. (Texto para Discussão).

TODD, P.; WOLPIN, K. On the especification and estimation of the production function for cognitive achievement. The Economic Journal, Oxford, v. 113, n. 485, p. F3-33, fev. 2003. https://doi.org/10.1111/14680297.00097

TOFFOLI, S. L. F.; SIMON, C. V. B. A utilização do modelo multifacetas de Rasch na análise das influências dos avaliadores sobre as avaliações com itens abertos. Ensaio: Avaliação e Políticas Públicas em Educação, Rio de Janeiro, v. 26, n. 101, p. 1303-23, out./nov. 2018. https://doi.org/10.1590/s010440362018002601146

TRAVITZKI, R. Enem: limites e possibilidades do exame nacional do ensino médio enquanto indicador de qualidade escolar. 2013. $322 \mathrm{f}$. Tese (Doutorado em Educação) - Universidade de São Paulo, Faculdade de Educação, São Paulo, SP, 2013.

WERLE, F. Políticas de avaliação em larga escala na educação básica: do controle de resultados à intervenção nos processos de operacionalização do ensino. Ensaio: Avaliação e Políticas Públicas em Educação, Rio de Janeiro, v. 19, n. 73, p. 769-92, out./dez. 2011. https://doi.org/10.1590/S0104-40362011000500003 


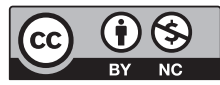

Rogério Severiano Dutra: Mestre em Administração e Controladoria pela Universidade Federal do Ceará. Contador do Instituto Federal de Educação, Ciência e Tecnologia do Ceará. Contato: rogerio.sdutra@yahoo.com.br

(iD) https://orcid.org/0000-0002-1352-1310

Giselle Bezerra Mesquita Dutra: Mestre em Educação pela Universidade Federal do Ceará. Professora da Secretaria de Educação do Estado do Ceará. Contato: gibmdutra@gmail.com (iD) https://orcid.org/0000-0001-6752-5870

Paulo Henrique Nobre Parente: Mestre em Administração e Controladoria pela Universidade Federal do Ceará. Professor do Instituto Federal de Educação, Ciência e Tecnologia do Ceará. Contato: paulonobreparente@gmail.com

Edilson Paulo: Doutor em Ciências Contábeis pela Faculdade de Economia, Administração e Contabilidade da Universidade de São Paulo. Pós-doutoramento em Controladoria e Contabilidade pela Universidade Federal de Santa Catarina. Professor Associado I da Universidade Federal do Rio Grande do Sul. Porto Alegre, Rio Grande do Sul, Brasil. Contato: edilson.paulo@ufrgs.br

(iD) https://orcid.org/0000-0003-4856-9039 